\title{
3D Weather - Towards a Real-time 3D Simulation of Localised Weather
}

\author{
Anthony Head \\ Bath School of Art and Design \\ Bath Spa University \\ Sion Hill, Bath \\ BA1 5SF \\ United Kingdom \\ www.3dweather.org \\ a.head@bathspa.ac.uk
}

\begin{abstract}
Weather forecasts are nearly always portrayed from either a satellite view perspective, a numerical or symbol based representation. None of these methods actually portray weather visually from the point of view of the observer, that is, they do not represent our experience of weather. This problem presents a challenge to displaying weather using real-time 3D computer graphics. 3D Weather is a proposed method to solve this problem, to create more believable representations of the weather using real weather data. By employing computer graphic techniques and computer game concepts the project intends to create a localized display of weather using mapping and weather data. Started in $\mathbf{2 0 1 0}$, the project has been exploring existing techniques, scoping out the needs of stakeholders (such as the Met Office), and creating a prototype to explore the issues. The paper concludes that the quest for realism with computer graphics can be a double-edged sword. It can lead to expectations of accuracy in the data its meant to represent, which can be desired, but in the case of the weather forecast the representation is not necessary what the weather will be, its what the weather might be. The continuing project will explore the balance of issues when representing the weather for past events as well as for forecasts.
\end{abstract}

Weather, 3D computer graphics, real-time, experience, virtual reality, simulation, forecast.

\section{INTRODUCTION}

For the last 10 years I have been engaged in 3D computer graphics for the purpose of art, involving weather and terrain simulation and virtual sculpture. Working with Jeremy Gardiner, we formed Light Years Projects (Head and Gardiner 2010) to create virtual artworks based on landscape subjects. This work has allowed me to study the nature of experience in a virtual painting as well studying the technical aspects of real-time computer graphics. One deliberate aspect of my work in projects such as Purbeck Light Years (Wands 2006) was not the pursuit of reality using computer graphics, but the pursuit of a painterly approach (Gardiner 2009). The virtual landscapes we created were based in part on data, but also on a much more subjective and emotive intention, through the use of paintings embedded into the work with the addition of sound.

It is from this background that I had thought about the issue of the representation of weather in the media and its relationship to people's experience of weather. Why doesn't the weather on the TV represent my experience of weather? I have proceeded to investigate this area for six months with specific aims.

\section{EXISTING WEATHER SIMULATION AND WEATHER FORECAST REPRESENTATION}

A vast majority of visual interpretation of weather forecasts is shown from a satellite or bird's eye viewpoint. This has the advantage of enabling the viewing of large areas of land (countries), whole weather systems and allowing broadcast to a large section of the population (i.e. a national or regional audience). Through these methods the science of weather and forecasts can be observed at the macro level. It has been an approach that serves communication media such as television broadcasting and newspaper publications. The disadvantage of delivering the information in this way is that there is no degree of personalisation allowed; the passive viewer cannot specify what information they wish to receive. 
The Internet has allowed advances in personalised weather forecast communication. Internet users can type in their location to receive weather forecasts relating to the location entered. However, this either takes the form of written data (e.g. temperature, description, pressure), or a close-up birds-eye view using two-dimensional graphics, either a still image or animated image (Met Office 2011).

The Internet allows the advantage of a personalised view of weather but still relies on the overhead satellite view in most cases (to show cloud positions), or numbers (such as temperature, pollen), or symbols (e.g. cloud, rain, sun). Companies such as the Met Office, employ a mix of all of these methods to express the scientific accuracy of their predictions, alongside past weather statistics.

These methods have the advantage that they can tell you accurate statistics, but the disadvantage that they do not represent the potential experience that the viewer might have if the predicted weather events occur. By not representing the user's potential experience, the method is less effective at convincing the user of the potential severity of weather events. I suggest that these methods are also not particularly memorable, as they do not affect you on an emotional level. Proof of this point will feature in the later stages of my project, with my initial investigation starting to address the issues.

One overall question of my project is: 'What methods can be employed to make a weather forecast more memorable and more believable than existing techniques?'

\subsection{First-person perspective weather}

\subsubsection{Internet based}

There have been attempts to show the weather in a different perspective to bird's eye view. Yo Window, (Repkasoft 2009-11) is such an application that uses weather data from the Norwegian Meteorological Institute (NMI) and a view of a generic landscape. By accessing weather data from the NMI, it provides visual representations of the weather from a fixed first-person perspective. Rain, clouds, snow, wind are all shown with varying degrees of change. Additionally, the weather statistics are shown as well. The application attempts to provide a more visually representative view of the weather. The location of your desired forecast can be entered, and the application responds by displaying the appropriate conditions.

However, also the weather conditions change, clouds appear or rain falls, the scenery stays the same. Also, there is not much subtlety in the weather that what is shown. For example, the difference between a shower starting and the rain in full flow is more of an off and on, rather than a gradual increase in rain. The systems show shower, rain or heavy rain.

So this lack of subtlety, combined with the fixed view of a stereotypical American farm leaves the viewer, in my opinion, not much better off than with the statistic and weather symbols. However, the ability to be able to slide through time does add a level of engagement and control. And it is possible that this view of the weather (a posterised vector image), does provide a more memorable and believable representation than just satellite views and statistics. It is potentially more fun.

\subsubsection{Game based}

The most commonly viewed first-person representations of weather are in computer games. Since the rise of 3D computers graphics, in particular for real-time gaming experiences, weather has been portrayed to add a sense of realism and atmosphere to the scene.

This paper is not going to examine all the computer games that have featured weather in. But I will mention some particular developments.

F1 $2010^{\mathrm{TM}}$ Game by Codemasters is a racing game featuring the tracks involved in the Formula 1 Grand Prix. It features weather (not linked to live data) that changes for each track. This included rain, and puddles, which affect how the car drives. The puddles are a particularly innovative feature, which appear as textures and show the reflections. The addition of water spray from the cars forming mists adds a convincing layer of accuracy to the way immersion of the player.

Microsoft Flight Simulator $\mathrm{X}$ is the latest version of the long running series of flight simulators. Weather has been a major feature of these games too, with clouds appearing in 3D based on weather settings. There is a plug-in that allows forecast data to be used by the game to display its clouds and rain. Time of day affects the colour of the sky too. The clouds are created by some of the processes described next in this paper. They feature several cloud forms, cumulus, stratus etc. The game player can fly through the clouds or view them from above and below.

\section{COMPUTER GRAPHIC METHODS FOR SIMULATING WEATHER}

For many years, computer scientists and programmers have been exploring methods of 
representing weather using 3D graphics, such as, the planets animation of James Blinn (Blinn 1982) 30 years ago. These techniques have ultimately fed into the development of real-time graphics for games, some of which l've just described.

\subsection{Clouds}

Naturally, the biggest visual elements of weather are clouds, and this area has formed a majority of efforts for technical study. There has been have been many different approaches, which fall into two classes: non-real-time rendered graphics and realtime graphics.

\subsubsection{Fluid dynamic approach}

Non-real-time methods generally aim to use highly mathematical methods to simulate the behaviour of weather events such as clouds. They follow fluiddynamic equations such as those attributed to Claude-Louis Navier and George Gabriel Stokes (Navier-Stokes equations) to create cloud formations, modelling the complete state of an enclosed system (i.e. a cloud). Indeed these are small scale versions that mirror how Meteorological organisations would use supercomputers to compute the projected path of whole weather systems, or indeed the whole Earth weather system. The masses of calculations needed to render clouds in this way means that current computing cannot display the results quickly enough. The user has to wait for a sequence to be rendered before playing it back.

A point to suggest here is that as computers get faster at processing, then the desire will be to show more complicated weather systems and hence processing time will keep increasing. The other issue with the highly mathematical approach to simulating weather is that dealing with a chaotic system, such as weather, means that there is inherent unpredictability. So mathematically predicting the actual shapes of clouds is probably impossible. However, types of clouds can certainly be represented using these methods.

\subsubsection{Real-time Methods}

Real-time methods for producing weather simulations have mainly focussed the following methods: metaballs, particle systems, impostors, voxels and cellular automata. In order to model the weather systems in real time (i.e. where the calculation of a frame takes a few milli-seconds), the calculation all of the physical detail of a cloud is not really possible (yet). There are several techniques that have been used to achieve the look of clouds, some of which can display a level of mathematical accuracy.

\subsubsection{Metaballs}

Metaballs, organic looking structures created from the proximity of points from several three dimensional geometric objects, have been used by many computer scientists to model clouds (e.g. Dobashi et al 1999). Used simply, the metaballs method would provide smooth surfaced clouds, but when enhanced with fractal blurs on the geometry points, they can create believable looking cloud structures. In their paper, Dobashi et al applied their technique to showing large weather systems based on satellite data. They even animated the clouds using wind direction vector data.

\subsubsection{Particle systems}

The 3D geometric method of particle systems takes advantage of the graphics processing unit's (GPU's) ability to render hundreds of thousands of points in milliseconds. With each particle displaying a small fluffy image. This has been used effectively to model individual and varied looking clouds (Schpok et al 2003). Small particles allow the possibility for using thermodynamic methods to display the clouds and, when applied with raytraced lighting, can created a believable looking result. However, re-calculating all particles to create a dynamic cloud in real-time, is a challenge. It can be done for a few clouds, but would cause problems when dealing with whole cloud systems as the calculations would slow down the frame-rate.

\subsubsection{Impostors}

The work of Mark Harris, summarised in the SIGGRAPH course of 2005 (Harris 2005), explains a range of techniques that can be employed to exploit the power of GPUs to display and calculate believable looking cloud formations.

Harris suggested using impostors (flat, large cloudlike particles). By using relatively simple ray-tracing methods applied to the imposters' colours, Harris was able to creating reasonably realistic cloud systems (groups of clouds) in 2002. Developments of these methods have been employed in Microsoft Flight Simulator (Wang 2003). This method is quick and, with optimising (reducing the number of impostors needed), complex systems of clouds can be produced.

\subsubsection{Voxels and cellular automata}

Voxels (3D pixels) allow for the possibility for a more a complex display of cloud structures. When using voxels, only the image that can be viewed by the camera is needed to be rendered. Replacing the voxel points with small blurred impostors can make the cloud solid looking (or translucent, as desired). Other parameters can be attached to the voxels (such as pressure, moisture), to allow a dynamic process to occur in the clouds. Work has been conducted in this area to create user defined sculpted clouds (Dobashi et al. 2008), that is clouds that form a shape drawn by the user. 
Earlier work by Dobashi and Nishita (Dobashi et al. 2000) focused on quick methods of creating dynamic clouds, using cellular automata. These methods allowed the physical dynamics of clouds to be created (not quite in real-time in 2000). The concept of approximating the physics led to a number of interesting results, including the modelling of clouds forming over mountains, and sunrays shining through clouds.

\subsection{Other weather elements}

My study so far has concentrated on clouds. But there are, of course, many other elements that make up the display of weather: rain, fog $/ \mathrm{mist} / \mathrm{haze}$, snow, wind, temperature, air pressure, snow, hail, rainbows, sunlight, heat haze etc.

I have previously used some of these methods in my Light Years Projects work. Light Years: Coast used particle systems for rain, with the rain's direction being affected by wind measurements. (Head and Gardiner 2010). 3D graphics commands allow simple fog settings to be altered and I have used these to vary the atmospheric perspective.

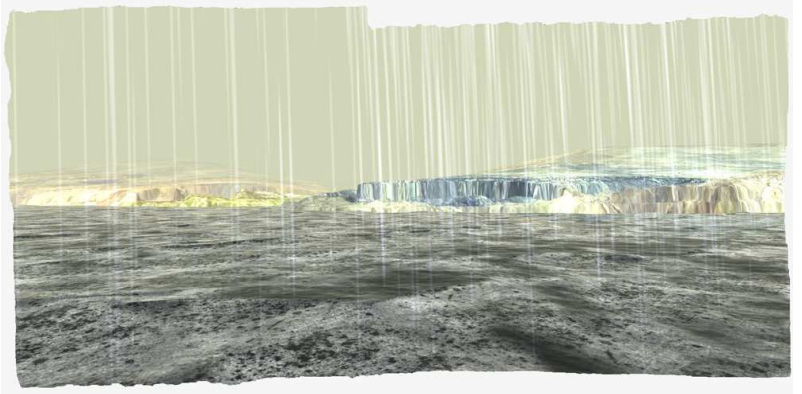

Figure 1 Light Years: Coast rain.

All of the previously listed weather elements are sky based, but one can also consider the effects of weather on the ground, e.g. puddles, laying snow, the effect of wind on trees/grass, splashes, shadows, light, ice, frost etc. Including these elements depends on a terrain. What that terrain should be leads to the question of the purpose of the simulation.

\section{A NEW WEATHER VIEWING APPLICATION}

My technical investigation has led me to create a prototype application. Using a set piece of terrain and photographic data, supplied by GetMapping Plc, l've been able to create a flythrough application. In the app, a randomised sample of a large satellite image is converted into cloud formations based on the clouds that are in the satellite image. The clouds do match the satellite images, although correct lighting of the clouds has yet to be implemented. Other issues, such as cloud base height and extrapolated multiple layers of clouds from the satellite images have yet to be resolved. The heights of clouds are calculated based on the cloud temperature gained from infrared satellite images.

The clouds appear volumetric, and a simple wind setting has been applied to demonstrate the movement of clouds across the landscape. Fog settings also simulate varying atmospheric perspectives based on the satellite image.

The terrain model has a $2 \mathrm{~m}$ posting from the original LIDAR information, and hence houses appear rounded, but the photography has a resolution of $12.5 \mathrm{~cm}$, which provides more visual detail. This helps distinguish the individual shape of the houses and is an important step towards the user being able to recognise their location. One limitation of this technique is that the photography is taken from a birds-eye view, and hence the sides of buildings display a stretched view of the roof, as opposed to windows and doors. Also, cars that feature in the photography aren't present in the terrain information, so they appear flat.

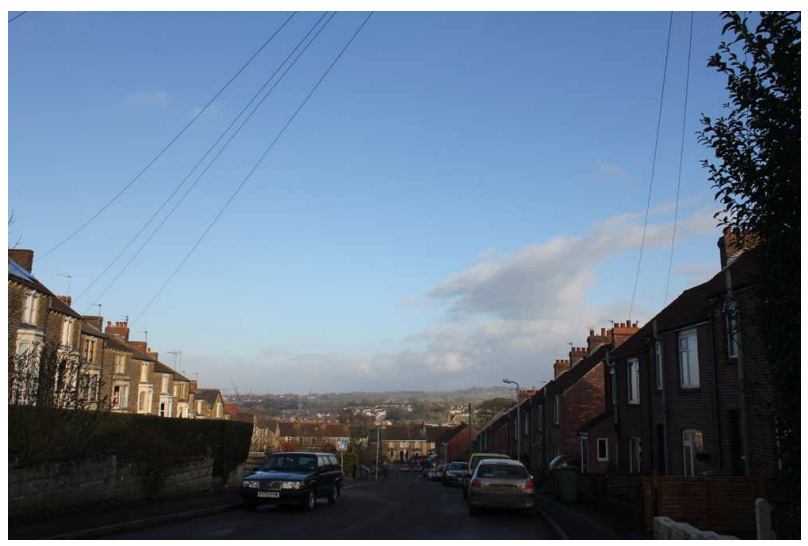

Figure 2a: A photograph of a view in Frome, Somerset, United Kingdom

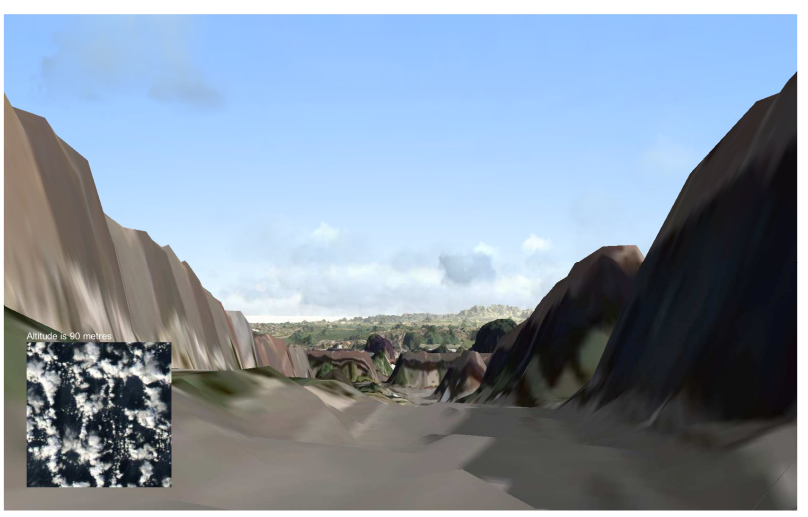

Figure 2b: 3D Weather representation of the same scene (but not with the correct clouds). 


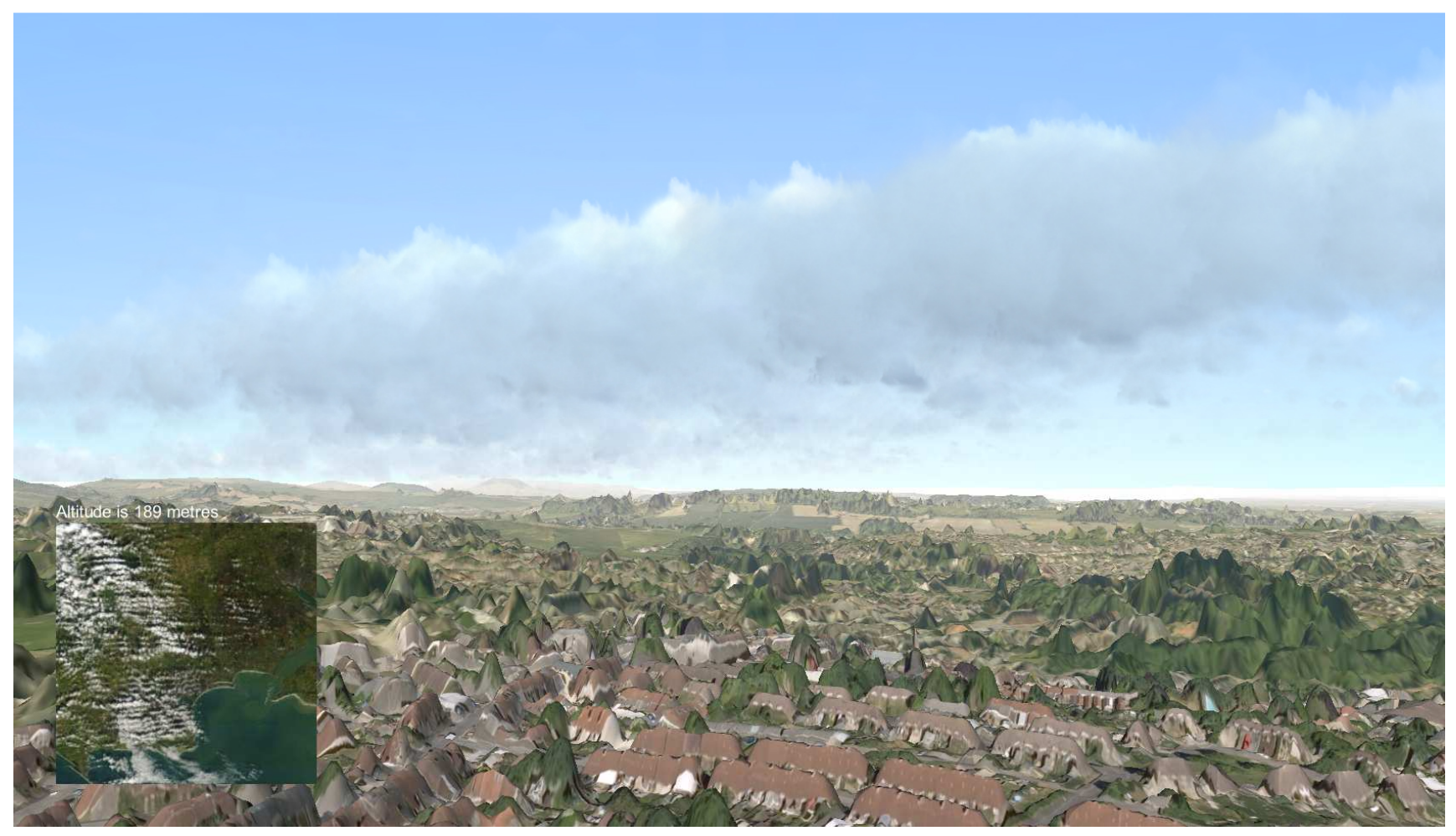

Figure 3: 3D Weather prototype showing terrain, clouds and a sample satellite image.

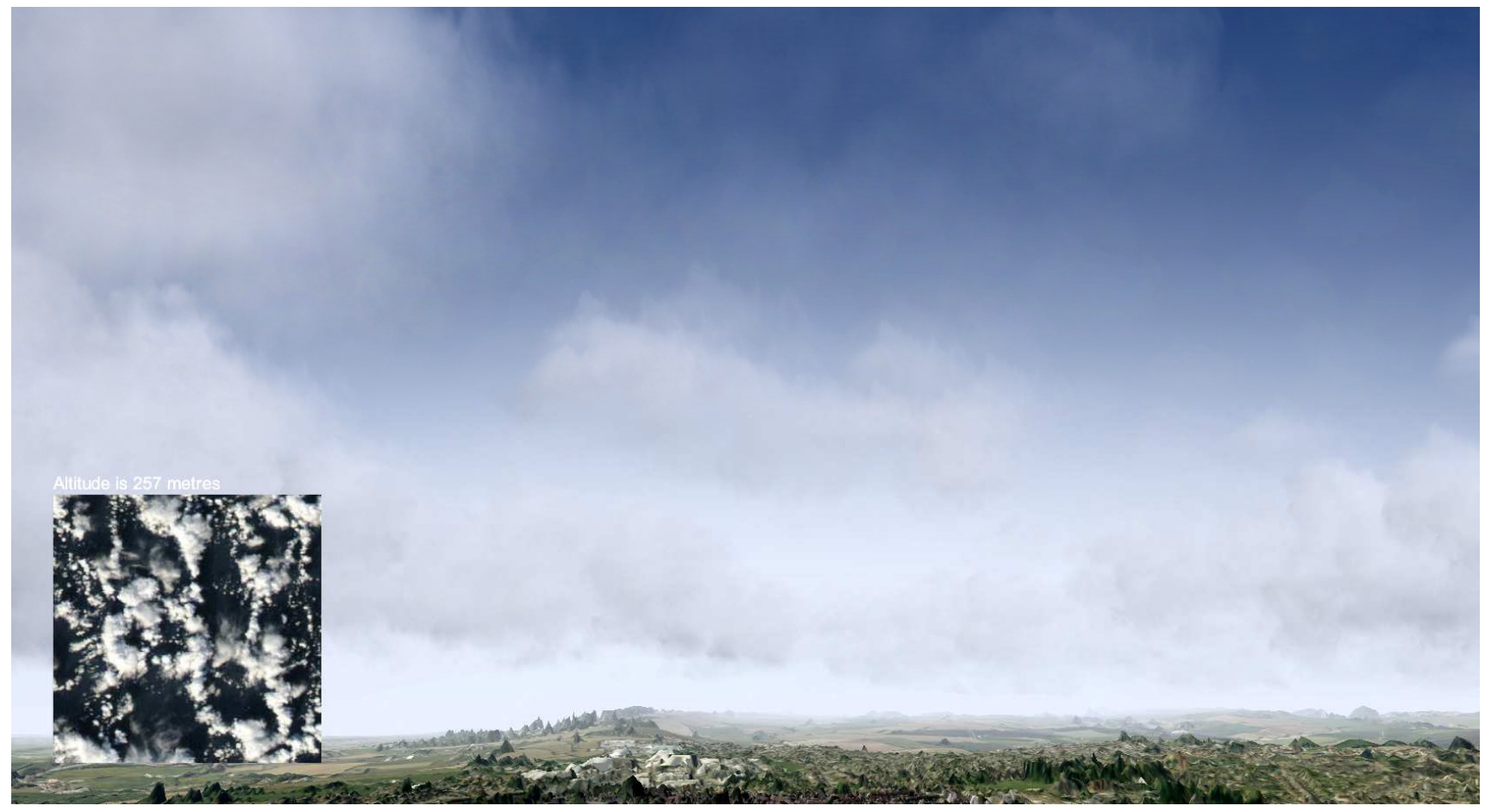

Figure 4: Another image from the 3D Weather prototype. 


\section{A QUESTION OF TRUTH, ACCURACY OR TRUST?}

The initial intention of the 3D Weather project was to create a believable 3D simulation of the weather that relates to the viewer's locality or chosen location. The reason I chose 3D graphics to do this is because I believe it offers the most adaptable methods for combining all of the variables needed to show the detail of the weather, in an instantly understandable manner. Parameters can be programmed to alter the image on screen in an infinite number of ways. This could allow me to take data and create accurate representations.

The above concept (if fully achievable) should be suitable for situations where scientific accuracy is needed. The 3D graphical display of the data could be tested against video or photographic evidence to see how representative it is. Thus an element of representation truth would be established in the simulation. The outcome of this is that the users could trust the system.

For an organisation like the Met Office, though, this is not necessarily their main objective. For scientists, the data they produce is what they need. However, the meteorological organisations create scientifically accurate data that they want to explain to the general public. In particular, they want to show that their weather predictions are based on scientific facts, but they do not want the public to believe that the predictions are scientific facts. However, the public often believe that forecasts are scientific facts. If the weather presenter says it is going to rain tomorrow then they are wrong if doesn't rain. This deterministic approach to forecasts can lead to these organisations' reputations being harmed, if they are wrong.

An alternative expression of the forecast is much more commonly used. The weather presenter will say that it might rain tomorrow or that there is a chance of rain. But this leaves the public not knowing, and in particular not having much confidence in what the presenter is saying. It is also not particularly scientific.

One approach that the meteorological organisations are keen on is the probabilistic forecast. For example "there will be a thirty per cent chance of rain tomorrow". This allows organisations such as events companies to act appropriately based on these kind figures. If there was a $90 \%$ chance of rain, they might cancel an event, if there is a $50 \%$ chance, they might carry on.

So where does this leave the idea of representing forecasts? The usual method is to use symbols and numbers, as well as satellite imagery. The $Y_{O}$ Window approach is to use the numbers, alongside simple representations of the clouds, and wind. What I question now is, whether the Yo Window approach is any different to showing symbols? The resolution of the representation (e.g. light cloud, heavy rain), is low and hence vague and the location visualisation does not relate to the viewers experience.

\section{CONCLUSION}

My interest in this subject came out of my interest in creating immersive environments, and a longterm interest in cloud representations. It is my belief in the engaging qualities of immersive environments that I propose the following: The more visually rich the environment the viewer is seeing, with visual cues to facts, then the more engaging the experience will be and more memorable the subtleties within the experience will be. The more engaging the experience then the more believable it is. Hence a greater resolution of data can and should be portrayed.

But given the issues described earlier about facts and forecasts, there is a careful path to tread.

So perhaps the aim for my investigation into weather representation should be this: to create a believable (in terms of location) representation of an environment whilst showing visually rich and memorable representations of weather. For displays of previous weather events the visual resolution of the clouds, rain etc, should and could be highly accurate.

There are a lot of subtleties in weather to display. And the approach could either to be use satellite images or to utilise textual descriptions. If I take snow for an example, snow words include: powder, slush, sleet, blizzard and flurry. Additional adjectives such as: light, heavy and nouns such as sprinkling, dusting, layer combine with these words to give many descriptions of snow. Further to this, measurements of snow depth and rate of snowfall tell us more information. This textual data could provide a high level of variation in how to show weather, without necessarily saying, there was specific cumulonimbus cloud that is pouring hail exactly on a specific location.

For displaying the forecast, there needs to be methods that emphasise the diminishing confidence or accuracy of that prediction as it goes further into the future. Thus the trust of the forecast simulation can be maintained. Utilising the textual descriptions might be the best way to show future weather, as they can be more vague, or open to interpretation. Whereas the accuracy of the satellite data, radar data and observed measurements would be more suitable for now-casting or the 
simulation of past weather. This point assumes that it is possible to confidently and accurately extract all of this data in a useful manner.

The next part of my investigation will cover areas that I now view as separate: forecast and past weather visual representation. Although they we initially intended to be achieved via the same approach, the issues that meteorologists face when sharing their information means that different factors come into play, when considering the audience.

\section{REFERENCES}

Blinn, J. (1982) Light reflection functions for simulation of clouds and dusty surfaces. SIGGRAPH '82 Proceedings of the 9th annual conference on Computer graphics and interactive techniques

Brickman, N., Olsen, D. \& Smith, G., Shapes in the Clouds: Interactive, Artist-Guided, Cloud Simulation. Methods.

Dobashi, Y., Nishita, T., Yamashita H., Okita T. (1999) Modeling of Clouds from Satellite Images Using Metaballs . Proceedings Pacific Graphics 98 Sixth Pacific Conference on Computer Graphics and Applications Cat No98 EX208. IEEE Comput. Soc, Pages: 53-60,

Dobashi, Y., Kusumoto, K., Nishita, T., and Yamamoto, T. (2008) Feedback control of cumuliform cloud formation based on computational fluid dynamics. ACM Transactions on Graphics, 27(3), p.1.
Dobashi, Y. et al., (2000) A simple, efficient method for realistic animation of clouds K. Akeley, ed. Proceedings of the 27th annual conference on Computer graphics and interactive techniques SIGGRAPH 00, p.19-28

Head, A. and Gardiner J. (2010) Light Years Projects. http://www.lightyearsprojects.org (26 March 2011)

Gardiner J. (2009) Light Years: Jurassic Coast. An immersive 3D Landscape Project. Proceedings from Electronic Visualisation and the Arts London. 2009.

Harris M. (2005) Real-time cloud simulation and rendering. SIGGRAPH '05. ACM SIGGRAPH 2005 Courses.

Met Office, (2011) UK Weather Forecast, http://www.metoffice.gov.uk/weather/uk/uk forecast weather.html (26 March 2011)

Repkasoft (2009-2011), Yo Window http://yowindow.com (Accessed 26 March 2011)

Schpok, J., Simons, J., Ebert, D. S. and Hansen, C. (2003) A Real-time Cloud Modeling, Rendering, and Animation system. In SCA '03: Proceedings of the 2003 ACM SIG- GRAPH/Eurographics Symposium on Computer Animation, pages 160166, Aire-la-Ville, Switzerland, Switzerland, 2003. Eurographics Association.

Wands, B. (2006) Art in the Digital Age. Thames and Hudson.

Wang, N. (2003) Realistic and fast cloud rendering in computer games. SIGGRAPH '03: ACM SIGGRAPH 2003 Sketches \& Applications, pages 1-1, New York, USA. ACM Press. 